\title{
Off-Label 9-Valent Human Papillomavirus Vaccination for Actinic Keratosis: A Case Series
}

\author{
Emily Wenande $^{\mathrm{a}} \quad$ Niels Bech-Thomsen ${ }^{\mathrm{b}} \quad$ Katrine Togsverd-Bo $^{\mathrm{a}}$ \\ Merete Haedersdal ${ }^{a}$ \\ aDepartment of Dermatology, Bispebjerg University Hospital, University of Copenhagen, \\ Copenhagen, Denmark; ${ }^{b}$ Clinic of Dermatology, Naestved, Denmark
}

\section{Keywords}

Human papillomavirus - Vaccine - Keratinocyte carcinoma - Actinic keratoses - Skin cancer .

Squamous cell carcinoma $\cdot$ Immunotherapy

\begin{abstract}
Background: The suspected link between human papillomavirus (HPV) and the development of premalignant and malignant skin lesions remains inadequately examined in clinical settings. This case series describes HPV vaccination as an off-label adjuvant therapy for actinic keratosis (AK). Methods: Twelve immunocompetent AK patients underwent HPV vaccination at a private dermatology clinic in Naestved, Denmark. Prior to vaccination, all patients demonstrated a high AK burden that required regular control visits. At 0,2, and 6 months, the patients received an intramuscular injection of a commercially available 9 -valent HPV vaccine. Concurrently, patients continued conventional AK therapies at 3-month intervals. Clinical response, consisting of reduction in AK number and general change in skin appearance, was assessed by a dermatologist over 12 months following first vaccination. Results: All patients (mean age 76.2 years; $10 \mathrm{M}$ and $2 \mathrm{~F}$ ) completed the vaccine schedule. Overall, an average $85 \%$ reduction in total AK burden was recorded 12 months after beginning vaccination. Median AK burden thus fell from 56 (IQR: 44-80) to 13.5 (IQR: 1-18) lesions after 12 months. Lesion reduction was observable by the second inoculation at month 2 (34 AKs; IQR 22-80), continuing steadily until month 6 (15 AKs; IQR 5-30) and plateauing between 6 and 12 months. Clinically, HPV vaccination elicited fading of lesions' erythematous background after the first dose, often followed by sloughing of hyperkeratotic elements in subsequent weeks. Patients reported no adverse effects related to HPV vaccination. Conclusion: This case series introduces the possibility that 9-valent HPV vaccination in combination with conventional treatments may be used as a therapeutic strategy for AK.
\end{abstract}


Table 1. Patient baseline characteristics

\begin{tabular}{|c|c|c|c|c|c|c|c|c|c|}
\hline \multirow[t]{2}{*}{ Patient } & \multirow[t]{2}{*}{ Sex } & \multirow[t]{2}{*}{ Age } & \multirow[t]{2}{*}{ Conventional AK therapy* } & \multirow[t]{2}{*}{ KC history $(n)$} & \multicolumn{5}{|c|}{ AKs per month visit $(n)$} \\
\hline & & & & & M0 & M2 & M6 & M9 & M12 \\
\hline 1 & $\mathrm{M}$ & 83 & CRYO, dPDT, rPDT, FU, IM & BCC (1) & 41 & 19 & 6 & 4 & 2 \\
\hline 2 & M & 80 & CRYO, DC & $\begin{array}{l}\text { SCC (7) } \\
\operatorname{BCC}(6)\end{array}$ & 83 & 94 & 47 & 19 & 15 \\
\hline 3 & M & 80 & CRYO, rPDT, IM, FU, DC & SCC (2) & 55 & 38 & 5 & 9 & 13 \\
\hline 4 & M & 78 & CRYO, rPDT, IM & BCC (4) & 51 & 35 & 17 & 5 & 0 \\
\hline 5 & M & 76 & CRYO, rPDT, IM, IMQ, DC & $\begin{array}{l}\text { SCC (3) } \\
\text { BCC (6) }\end{array}$ & 124 & 92 & 33 & 24 & 18 \\
\hline 6 & M & 75 & CRYO, rPDT, IMQ & BCC (6) & 33 & 26 & 3 & 0 & 0 \\
\hline 7 & M & 73 & CRYO, IMQ & BCC (1) & 57 & 11 & 5 & 0 & 1 \\
\hline 8 & M & 73 & CRYO, rPDT, IM & $\begin{array}{l}\text { SCC (1) } \\
\text { BCC (7) }\end{array}$ & 470 & 384 & 60 & 57 & 31 \\
\hline 9 & M & 72 & CRYO, rPDT, IM, IMQ & $\begin{array}{l}\text { SCC (3) } \\
\text { BCC (2) }\end{array}$ & 72 & 24 & 18 & 9 & 8 \\
\hline 10 & M & 70 & CRYO, dPDT & SCC (1) & 32 & 21 & 8 & 14 & 14 \\
\hline 11 & $\mathrm{~F}$ & 78 & CRYO, rPDT, FU & $\begin{array}{l}\text { SCC (2) } \\
\text { BCC (2) }\end{array}$ & 65 & 44 & 20 & 14 & 18 \\
\hline 12 & $\mathrm{~F}$ & 76 & CRYO, rPDT, FU, IM, IMQ & BCC (1) & 53 & 33 & 13 & 17 & 16 \\
\hline
\end{tabular}

AK, actinic keratosis; BCC, basal cell carcinoma; CRYO, cryotherapy; DC, diclofenac; dPDT, daylight photodynamic therapy; FU, 5-fluorouracil; IMQ, imiquimod; IM, ingenol mebutate; KC, keratinocyte carcinoma; rPDT, red light photodynamic therapy; SCC, squamous cell carcinoma.

*Therapies including in the past 12 months.

\section{Introduction}

An association between skin dysplasia and human papillomavirus (HPV) has long been suspected. Raising suspicion of a potential relationship, the prevalence of viral DNA and mRNA is reported in actinic keratoses (AKs) as well as keratinocyte carcinomas (KCs) [1-3]. Risk of skin cancer is higher in immunosuppressed populations, with HPV DNA detection approaching $90 \%$ in KCs of immunosuppressed versus 30-50\% in immunocompetent groups $[2,4]$. At present, no prospective clinical trial has examined the therapeutic potential of HPV vaccination for $\mathrm{KC}$ or its precursors [5]. In the following case series, we describe 9-valent HPV vaccination as an off-label, adjunctive treatment for AK.

\section{Case Series}

Between January 2019 and May 2020, 12 immunocompetent Caucasian patients (10 M and 2 F) were treated at a private dermatology clinic in Naestved, Denmark (Table 1). All patients had responded inadequately to previous conventional $\mathrm{AK}$ treatments and sought to minimize future need for destructive therapies. Patients furthermore all had a history of KC. Vaccination was offered on a case-by-case basis for selected, difficult-to-treat patients with high AK burden. Patients provided informed written consent to off-label treatment with HPV vaccine prior to initiating vaccination. 


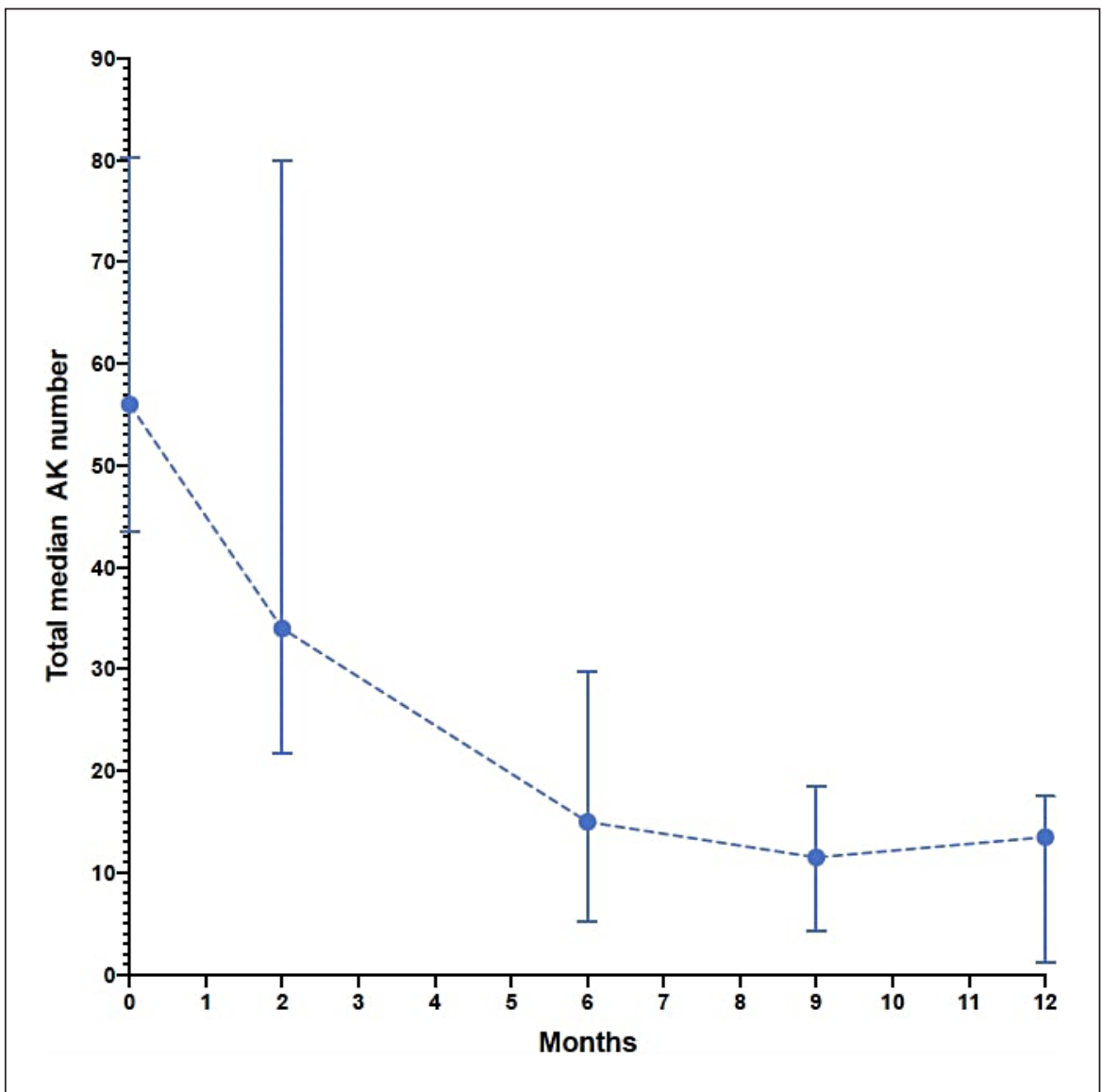

Fig. 1. Clinical reduction in AK burden after 9-valent HPV vaccination. Reduction in median total AK number over a 1-year observation period. Error bars represent interquartile ranges. Overall, an average $85 \%$ reduction in the total AK number is observed from baseline to 12 months. HPV, human papillomavirus; AK, actinic keratosis.

According to the standard vaccination schedule, individuals received an intramuscular injection of a commercially available 9 -valent HPV vaccine (GARDASIL ${ }^{\circledR}$; Merck \& Co, Whitehouse Station, NJ, USA) at 0,2 , and 6 months. Patients continued individualized AK management with conventional therapies at 3-month intervals, consisting of lesion-directed cryotherapy and field-directed photodynamic therapy (Table 1). No patients initiated new AK treatments after the first vaccination. All AKs on the head/face, trunk, and extremities were counted during visits, providing a total lesion count at month 0,2 , and 6 (vaccination visits) as well as 9 and 12 (routine control visits).

Prior to vaccination, AK burdens ranged from 32 to 470 lesions (median: 56; interquartile range [IQR] 44-80). Overall, AK burden showed marked decline from baseline lesion count (56 AKs; IQR: 44-80) (Fig. 1). At the 2-month vaccination visit, the total AK number thus dropped an average of 35\% (34 AKs; IQR: 22-80). By the third vaccination visit at 6 months, a 77\% decline in AKs was noted (15 AKs; IQR: 5-30). AK burden appeared to stabilize between 6 and 12 months, ultimately resulting in a median lesion count of 13.5 (IQR: 1-18). Thus, a year after beginning HPV vaccination, total AK count was $85 \%$ lower than baseline.

Clinical responses were discernible within 2 months of initiating vaccination. Thick AKs flaked off, leaving flat, scaly, and less erythematous lesions. Similarly in thin AKs, the 

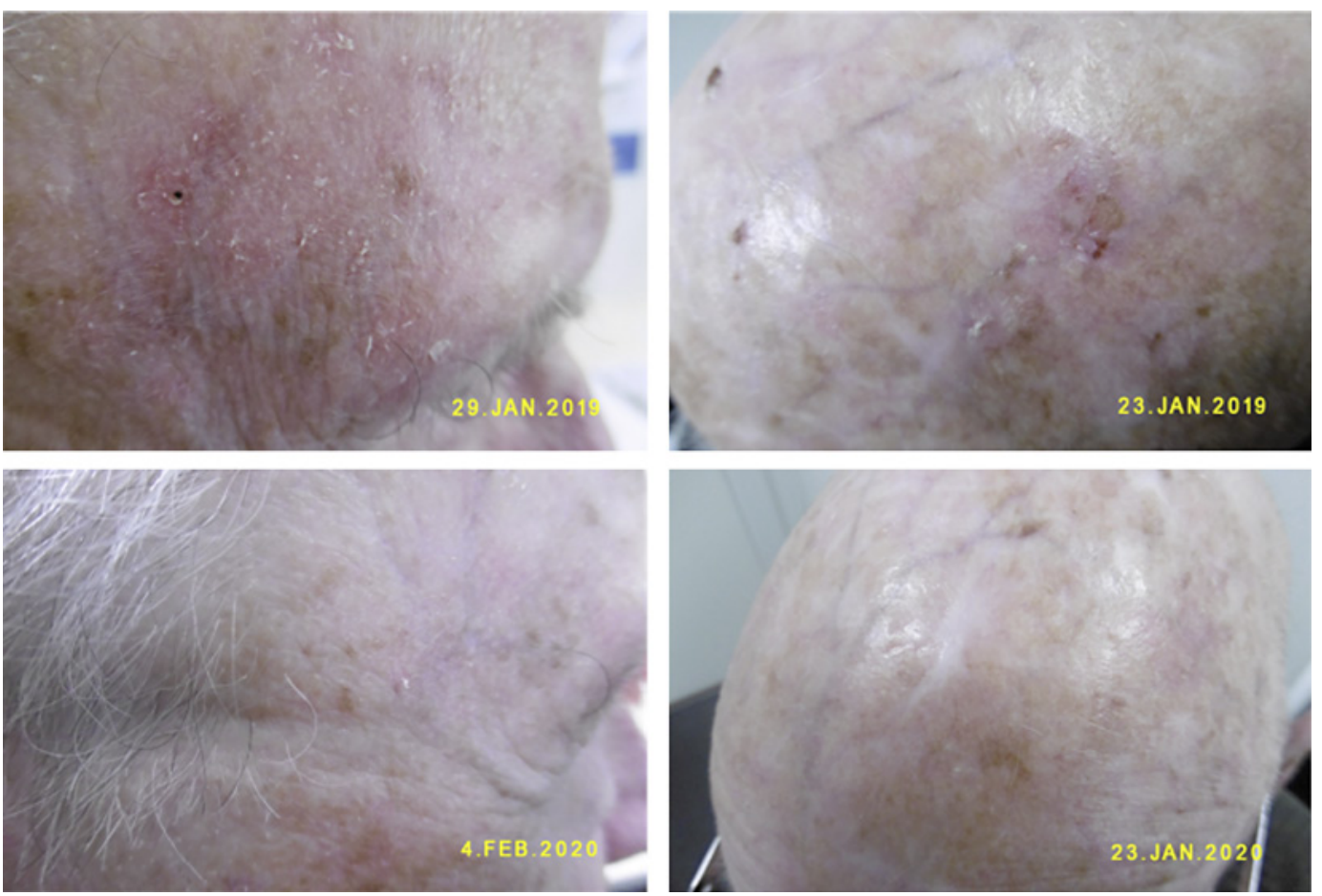

Fig. 2. Clinical response to HPV vaccination documented by digital photography. Clinical AK clearance and improvement in skin texture is pictured at baseline and 12-month follow-up in 2 patients after adjunctive HPV vaccination. Visible reduction in AK lesions is observed in both instances. HPV, human papillomavirus; $\mathrm{AK}$, actinic keratosis.

erythematous background appeared to fade (Fig. 2), presenting in dermoscopy as a disappearance of the characteristic red pseudo-network.

\section{Discussion}

This case series describes a possible therapeutic effect of 9-valent HPV vaccination for skin cancer precursors. In the literature, there are emerging reports of the vaccine's potential as an off-label treatment strategy for HPV-associated cutaneous conditions [5]. Indeed, using an identical vaccination protocol, we previously treated a patient with exceedingly high AK burden $(>1,000 \mathrm{AKs})$, noting marked reduction in lesion number and improved skin texture over 1 year [6]. Interestingly, systemic and intratumoral use of HPV vaccines were recently described in the context of KC for 4 immunocompetent and 1 immunocompromised patient [7-10]. In comparison with KCs, an accelerated response seemed to occur in precursor AKs. Thus, clinical AK regression typically began within 2 months of the first injection, resulting in significant AK clearance before the vaccination protocol was completed. The higher viral load reported for AKs versus KC lesions may explain this potentiated response [1].

Based on our 1-year clinical observation, adjunctive HPV vaccination appeared beneficial for this selected patient profile, as reflected by a reduction in total AK burden in all patients versus baseline. Importantly, conventional treatments previously administered at regular 3 -month visits with equal aggressiveness had failed to achieve comparable reductions in patients' AK burden. We thus interpret HPV vaccination to have contributed to the drop in AK numbers. Devoted sun-worshippers and patients with many AKs on the scalp, face, and neck 
were initially slower to respond to HPV vaccination. Furthermore, a patient that during the 1-year period developed unrelated, life-threatening illness experienced a transient increase in AKs at the 6-month visit. Increased sun exposure and immune compromise may represent negative predictors of vaccination response.

The mechanism behind HPV vaccine-mediated effects on AKs and KCs is unknown. Previously, $\beta$-HPVs were theorized to play a role in the initiation phase of skin carcinogenesis, possibly via the deleterious effects of viral oncoproteins, E6 and E7 [3, 11-13]. In light of this theory, the question remains why a vaccine targeting mucosal $\alpha$-HPVs would provide nearuniversal effects in our AK population. Among the vaccine-associated types, HPV16 is only occasionally detected in skin lesions [3]. AKs instead primarily harbor $\beta$-HPVs, which in turn exhibit marked variation in type between lesions and individuals [14]. Rather than causative, the link between HPV and skin cancer may be correlative. Strickley et al. [15] recently showed that the $\mathrm{CD}^{+} \mathrm{T}$-cell immune response against HPV, stimulated by infection, vaccine, or T-cell transfer, suppresses skin cancer development in mice. The authors propose that proliferating keratinocytes (i.e., in pre/malignant lesions) harboring active HPV can be targeted through boosts in HPV immunity and activation of T cells residing in the skin [15]. This mechanism may explain the clinical effects observed in our immunocompetent population, where some but not all AKs might be expected to harbor HPV [2]. AK reduction could further be driven by immunological responses generated by joint stimulation of vaccination and local immune cell recruitment by continued local therapies.

This small case series constitutes a preliminary description of the clinical effect of adjuvant 9-valent HPV vaccination in a small group of immunocompetent patients with high AK burden, treated at a private dermatology practice in Denmark. Crucially, as is the nature of a case series, there is no control group receiving comparable conventional therapy alone. It is therefore unclear to what degree vaccination contributed to the observed responses. We can furthermore not confirm that lesion clearance is sustained beyond a year. Nevertheless, we found that the dramatic and rapid response observed here in 12 patients warrants attention. This case series thus aims to inspire larger, randomized controlled trials investigating the efficacy and underlying mechanism behind HPV vaccination for AK.

\section{Statement of Ethics}

In this series of cases, all patients provided written informed consent to receive off-label vaccination with the 9-valent human papillomavirus (HPV) vaccine prior to initiating the treatment, as well as to publication of this report and accompanying images. Since vaccination was offered at private dermatology practice on a case-by-case basis to selected, difficult-to-treat patients with high AK burden, approval by an international review board was not sought.

\section{Conflict of Interest Statement}

E. Wenande, N.B. Thomsen, K. Togsverd-Bo, and M. Haedersdal have no conflicts of interest to declare. The authors have no relevant financial activities outside the submitted work or during the 3 years prior to submission. All authors have no financial or nonfinancial (political/personal) interests, relationships, or affiliations relevant to the subject of the manuscript. Specifically, the commercial manufacturer of the 9-valent HPV vaccine has neither been informed nor financially involved in the planning, conduction, or reporting of this case series. The authors have no relevant conflicts of interest in regard to employment, stock,

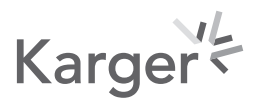


royalties, or consultant arrangements with any HPV vaccine manufacturer. The manuscript's contents have not previously been presented elsewhere.

\section{Funding Sources}

No external funding went toward the planning, execution, or writing of this report. Vaccines were purchased by Dr. N.B. Thomsen's private dermatology practice and provided free of charge to all patients included in this report.

\section{Author Contributions}

All authors contributed to the treatment concept. N.B. Thomsen performed all patient treatments and collection of raw data. E. Wenande had full access to all study data and takes responsibility for the accuracy of the data analysis. E. Wenande performed the data analysis and prepared the manuscript in collaboration with K. Togsverd-Bo and M. Haedersdal.

\section{Data Availability Statement}

All data, with the exception of nonpublished patient images, generated or analyzed during this study are included in this article. Further enquiries can be directed to the corresponding author.

\section{References}

1 Weissenborn SJ, Nindl I, Purdie K, Harwood C, Proby C, Breuer J, et al. Human papillomavirus-DNA loads in actinic keratoses exceed those in non-melanoma skin cancers. J Invest Dermatol. 2005;125(1):93-7.

2 Harwood CA, Surentheran T, McGregor JM, Spink PJ, Leigh IM, Breuer J, et al. Human papillomavirus infection and non-melanoma skin cancer in immunosuppressed and immunocompetent individuals. J Med Virol. 2000; 61(3):289-97.

3 Ben Ayed I, Tounsi H, Jaballah A, Ardhaoui M, Maaloul A, Lassili T, et al. Mucosal human papillomavirus detection and TP53 immunohistochemical expression in non-melanoma skin cancer in Tunisian patients. J Cutan Pathol. 2019;46(8):591-8.

4 Borgogna C, Lanfredini S, Peretti A, De Andrea M, Zavattaro E, Colombo E, et al. Improved detection reveals active $\beta$-papillomavirus infection in skin lesions from kidney transplant recipients. Mod Pathol. 2014;27(8): 1101-15.

5 Pham CT, Juhasz M, Sung CT, Mesinkovska NA. The human papillomavirus vaccine as a treatment for human papillomavirus-related dysplastic and neoplastic conditions: a literature review. J Am Acad Dermatol. 2020; 82(1):202-12.

6 Wenande E, Bech-Thomsen N, Haedersdal M. Reduction in actinic keratoses following 9-valent human papilloma virus vaccination. Dermatol Ther. 2020;33(4):e13454.

7 Nichols AJ, Allen AH, Shareef S, Badiavas EV, Kirsner RS, Ioannides T. Association of human papillomavirus vaccine with the development of keratinocyte carcinomas. JAMA Dermatol. 2017;153(6):571-4.

8 Nichols AJ, De Bedout V, Fayne RA, Burke GW, Kirsner RS, Ioannides T. Systemic and intratumoral 9-valent human papillomavirus vaccine treatment for squamous cell carcinoma in situ in a renal transplant recipient. JAAD Case Rep. 2020;6(4):289-91.

9 Nichols AJ, Gonzalez A, Clark ES, Khan WN, Rosen AC, Guzman W, et al. Combined systemic and intratumoral administration of human papillomavirus vaccine to treat multiple cutaneous basaloid squamous cell carcinomas. JAMA Dermatol. 2018;154(8):927-30.

10 Geizhals SLMG. The successful treatment of multiple cutaneous malignancies with HPV vaccination: case report. SKIN J Cutan Med. 2020;4(2):148-51.

11 Conforti C, Paolini F, Venuti A, Dianzani C, Zalaudek I. The detection rate of human papillomavirus in welldifferentiated squamous cell carcinoma and keratoacanthoma: is there new evidence for a viral pathogenesis of keratoacanthoma? Br J Dermatol. 2019;181(6):1309-11. 
12 Drvar DL, Lipozenčić J, Sabol I, Mokos ZB, Ilic I, Grce M. Human papillomavirus status in extragenital nonmelanoma skin cancers. Clin Dermatol. 2014;32(2):248-52.

13 Accardi R, Gheit T. Cutaneous HPV and skin cancer. Presse Med. 2014;43(12 Pt 2):e435-43.

14 Correa RM, Vladimirsky S, Heideman DA, Coringrato M, Abeldaño A, Olivares L, et al. Cutaneous human papillomavirus genotypes in different kinds of skin lesions in Argentina. J Med Virol. 2017;89(2):352-7.

15 Strickley JD, Messerschmidt JL, Awad ME, Li T, Hasegawa T, Ha DT, et al. Immunity to commensal papillomaviruses protects against skin cancer. Nature. 2019;575(7783):519-22. 